\begin{tabular}{|c|c|c|}
\hline Beitr. Ent. & Keltern & ISSN 0005-805X \\
\hline $\mathbf{6 0}(2010) 1$ & S. $71-79$ & 31.07 .2010 \\
\hline
\end{tabular}

\title{
Zum Vorkommen der auf Säugetieren parasitierenden Lausfliegen in der Uckermark
}

\section{(Diptera, Hippoboscidae)}

Mit 2 Figuren und 1 Tabelle

Matthias Jentzsch \& Frank Menzel

\begin{abstract}
Zusammenfassung
An 20 verschiedenen Wald-Standorten wurden in der Uckermark-Region (Nordost-Deutschland) Lausfliegen (Hippoboscidae) gesammelt. Das Material stammt aus Dipteren-Proben, die in den Jahren 2001 bis 2003 im Rahmen des Forschungsprojektes »Nordmitteleuropa 2020« (NME2020) gekeschert wurden. Im Ergebnis dessen sind die Lausfliegen-Arten Hippobosca equina Linnaeus, 1758 und Lipoptena cervi (Linnaeus, 1758) in der Uckermark nachgewiesen worden. Dabei handelt es sich ausschließlich um geflügelte Weibchen, die wahrscheinlich auf Wirtssuche waren. Beide Arten parasitieren in der Regel bei großen Säugetieren (H. equina vor allem beim Hauspferd, L. cervi vor allem beim Reh), sind allgemein häufig und weit verbreitet. Die vorgestellten Nachweise werden als Beiträge zur Lausfliegen-Fauna der Bundesländer Brandenburg und Mecklenburg-Vorpommern betrachtet.
\end{abstract}

\section{Summary}

Louse flies (Hippoboscidae) were collected from 20 different wooded areas in the Uckermark region (north-east Germany). The specimens were taken from Diptera samples caught during a reseach project "Nordmitteleuropa 2020« (NME2020) in 2001 to 2003. As a result of this, the louse flies Hippobosca equina Linnaeus, 1758 and Lipoptena cervi (Linnaeus, 1758) were identified from the Uckermark. Only females were found and they were exclusively winged and probably seeking vectors. Both species generally parasitise large mammals (H. equina mainly domestic horses, L. cervi mainly deer) and both species are usually common and widespread. This evidence makes a valuable contribution to the louse fly fauna of Brandenburg and Mecklenburg-Vorpommern.

Key words

Diptera, Hippoboscidae, Germany, Brandenburg, Mecklenburg-Vorpommern, Uckermark, Ucker, faunistics, new records

\section{Einleitung}

Von den weltweit 204 Arten der Hippoboscidae kommen in Deutschland nur 15 Spezies vor. In der Checkliste der Dipteren Deutschlands (Müller 1999) sind 12 Spezies verzeichnet. Darauf aufbauend wurden später durch Коск (2000), MülLER (2000b), SCHMidt (2001) und Müller \& Schмidт (2001) diefolgenden 3 Arten mit Funden in Hessen, Baden-Württemberg und Thüringen hinzugefügt: Drossellausfliege - Ornithioca turdi (LATreIlle, 1812), Alpenseglerlausfliege Crataerina melbae (RoNDANI, 1879) und Reiherlausfliege - Icosta ardeae (MACQUART, 1835). 
Die Hirschlausfliege - Lipoptena cervi (Linnaeus, 1758) - besitzt ein oligoxenes Wirtsspektrum, ist in der gesamten Paläarktis weit verbreitet und in Mitteleuropa einer der häufigsten Vertreter (BüTtiker 1994). In der Regel werden größere Säugetiere - überwiegend Rehe (Capreolus capreolus) - befallen. Es sind aber auch andere Wirte, wie z. B. Amsel (Turdus merula), Igel (Erinaceus europaeus), Damhirsch (Dama dama), Haushund (Canis lupus f. familis) und der Mensch (Homo sapiens) bekannt (MülleR 1985, 1997).

Ebenfalls in Mitteleuropa sehr häufig und in der paläarktischen Region weit verbreitet ist die Pferdelausfliege (Hippobosca equina Linnaeus, 1758), die auch in der Afrotropis und Orientalis vorkommt. Sie lebt relativ monoxen auf Pferden (Equus caballus), tritt selten aber auch beim Hausrind (Bos taurus domesticus) oder beim Menschen auf (BüTTIKer 1994). MüLler (2009) nennt als weiteren Wirt den Hausesel (Equus asinus f. familis). In Deutschland ist über die lokale Verbreitung von Hippobosca equina jedoch relativ wenig bekannt.

Lausfliegen lassen sich durch andere, größere Tiere transportieren (Phoresie). Vor allem bei Zugvögeln ist der Nachweis von Hippobosciden schwierig und unterliegt meist dem Zufall. Darum lassen sich fundierte Aussagen zur Verbreitung und Ausbreitungsdynamik der Lausfliegenspezies nur schwer treffen. Für Lipoptena cervi - mit Präferenz für terrestrische Säugetiere - ist das weniger problematisch. Bei Hippobosca equina kommt jedoch noch der Aspekt der anthropogen bedingten Ausbreitung hinzu (z. B. durch Haustiertransporte).

Die hier betrachteten Lausfliegen wurden im Rahmen des ZALF-Verbundprojektes "Nachhaltige Landschaftsentwicklung - Nordmitteleuropa 2020« (Teilprojekt III: Biotische Integrität der Agrarlandschaften) gesammelt. In den Folgejahren hat man die dipterologischen Untersuchungen in den Projekt-Titel "Charakterisierung der biotischen Naturraumausstattung " des Programmbereiches 3 (Biodiversität und Lebensraumfunktionen in der nachhaltigen Landschaftsentwicklung) überführt. Vordergründig sind die Freilandarbeiten des Deutschen Entomologischen Institutes [heute: Senckenberg Deutsches Entomologisches Institut (SDEI), Müncheberg] mit dem Ziel durchgeführt worden, einen Beitrag zur Diversität von Trauermücken (Sciaroidea: Sciaridae) zu leisten und deren bioindikatorische Eignung zu untersuchen. Im Gelände wurden aber auch andere Dipterengruppen aus den Proben extrahiert und ausgewählten Spezialisten zur Bearbeitung übergeben. Zu den Beifängen gehören nicht nur die Hippoboscidae, sondern v. a. Mücken und Fliegen aus den artenreichen Überfamilien der Sciaroidea (Bolitophilidae, Diadocidiidae, Ditomyiidae, Keroplatidae, Mycetophilidae) und der Empidoidea (Dolichopodidae, Empididae, Hybotidae). Die Trauermücken- und Tanzfliegenverwandten können als Nahrungskonkurrenten oder als potentielle Räuber einen Einfluß auf die Sciaridenzönose haben. Aus diesem Grund sind die neuen Daten nicht nur für die »Entomofauna Germanica « bedeutsam und runden unseren Kenntnisstand zum Vorkommen der Zweiflügler ab, sondern sie liefern auch wichtige Informationen zum Beziehungsgefüge in den untersuchten Waldökosystemen. Im ersten Beitrag stellen wir zunächst die Lausfliegen-Funde vor, die sich in den Jahren 2001 bis 2003 im Einzugsbebiet der Ucker (Nordostdeutsches Tiefland) ergeben haben.

\section{Untersuchungsgebiet}

Für die dipterologischen Untersuchungen wurde das Einzugsgebiet des Flusses Ucker (= Uecker) ausgewählt. Die Ucker entspringt bei ca. $70 \mathrm{~m}$ ü. NN in der Gemeinde Temmen-Ringenwalde (Landkreis Uckermark). Der Fluß ist 103 km lang und mündet in das Stettiner Haff. Auf seinem Weg nach Norden werden die Bundesländer Brandenburg und Mecklenburg-Vorpommern berührt sowie die Städte Prenzlau, Pasewalk, Torgelow und Ückermünde passiert.

${ }^{1}$ ZALF = Leibniz-Zentrum für Agrarlandschaftsforschung Müncheberg 
Das Ucker-Einzugsgebiet wird im Norden vom Stettiner Haff und im Osten durch die Oder (zugleich Staatsgrenze zur Republik Polen) begrenzt. Im Westen reicht das Ucker-Einzugsgebiet nur wenig über die gedachte Linie Ückermünde - Strasburg - Feldberg hinaus. In Nord-Süd-Richtung liegen an der nordwestlichen Grenze zunächst die "Große Friedländer Wiese" (E von Friedland) und die "Brohmer Berge« (N von Strasburg). Im Südwesten wird die beprobte Fläche von ausgedehnten Schutzgebieten eingerahmt. Sie reicht von den östlichen Ausläufern des Naturparks "Feldberger Seenlandschaft" nahe Feldberg, über den Naturpark "Uckermärkische Seen" nördlich von Templin bis zum "Biosphärenreservat Schorfheide-Chorin « nördlich von Joachimsthal. Die südlichste Ausdehnung erreicht das Untersuchungsgebiet etwa in Höhe der Verbindungslinie Templin - Angermünde. Der südwestliche Zipfel wird von den Ortschaften Milmersdorf (E von Templin), Ringenwalde und Steinhöfel (W von Greiffenberg) begrenzt. Von dort bildet - mit Ausnahme eines kleinen Abschnittes zwischen Schmölln und Storkow - die Autobahn von Berlin nach Szczecin (A11) bis zum Grenzübergang Pomellen/Kołbaskowo die südöstliche Begrenzung. Das Einzugsgebiet der Ucker (Fig. 1) nimmt eine Fläche von $2.426 \mathrm{~km}^{2}$ ein. In der glazial überformten Kulturlandschaft liegt der Anteil der Waldflächen bei ca. 20 \%. Diese bestehen etwa zu $60 \%$ aus Nadelwäldern (meist Kiefer) und $40 \%$ aus Laubwäldern (meist Buche). Nach diversen Waldumbaumaßnahmen bilden sich zunehmend auch Mischwälder heraus. Im Gebiet herrschen sandige bis lehmige Böden (94 \%) vor. Die Jahresdurchschnittswerte (1970-1999) für Temperatur und Niederschlag werden mit $+8,2^{\circ} \mathrm{C}$ und $552 \mathrm{~mm}$ pro Jahr angegeben.

\section{Material und Methoden}

Für das Projekt wurden 50 Waldstandorte mit unterschiedlicher Biotopstruktur ausgewählt (Fig. 1), so daß sich daraus eine relativ konstante Vernetzung über das gesamte Gebiet ergibt. Einige Untersuchungsflächen (Fundpunkte 3, 5, 6, 7, 14, 40, 42 und 44) liegen etwas außerhalb des beschriebenen Gebietes. Diese wurden in das Arbeitsprogramm aufgenommen, weil sich in einigen geographischen Räumen entlang der definierten Grenzen des Ucker-Einzugsgebietes keine geeigneten Waldbiotope befinden. Im ersten Versuchsjahr wurden die Flächen ausgewählt, die standortspezifischen Daten ermittelt und die untersuchten Waldbiotope charakterisiert. Im Ergebnis dessen liegen für alle Waldstandorte (Fundpunkte 1-50) detaillierte Informationen zu den Koordinaten, der Höhe ü. NN, dem ph-Wert des Bodens und den vorgefundenen Laubund Nadelgehölzen vor. Außerdem wurde eine umfangreiche Fotodokumentation von den Versuchsflächen angelegt.

In dem beschriebenen Gebiet fanden von Anfang Juli 2001 bis Ende September 2003 großflächige Untersuchungen mit der Kescherstreifmethode statt. Gekeschert wurde in unmittelbarer Nähe der Bodenauflage, in bzw. über der Krautschicht und an Blättern von niedrigen Sträuchern. Einbezogen wurden auch morsche oder bepilzte Baumstubben, Fallholz-Ansammlungen und aufgeklappte Wurzelteller. Die Probenentnahme erfolgte mit einem Netzkescher $(33$ x $36 \mathrm{~cm})$, bei dem das $70 \mathrm{~cm}$ lange Gazenetz durch einen Leinenbeutel geschützt wird. Nach je 25 Doppelschlägen wurde der Kescherinhalt nach den Zielgruppen abgesucht und die Imagines mit dem Exhaustor entnommen. Das Abtöten der Dipteren erfolgte mit Essigether in den auswechselbaren Exhaustorflaschen $(\mathrm{H}=9 \mathrm{~cm} / \varnothing=4,5 \mathrm{~cm})$. Vor dem Verlassen der Versuchsfläche wurden die Proben in unvergällten, 70 \%igen Alkohol überführt und im Labor mit dem Binokular nach Familien vorsortiert.

Alle Waldstandorte wurden einmal im Jahr aufgesucht und standardisiert mit 600 Kescherschlägen beprobt. Zu diesem Zweck ist das Untersuchungsgebiet in drei, etwa gleichgroße Flächen - einen Nord-, Südwest- und Südost-Sektor - aufgeteilt worden. Bei geeigneten Witterungsverhältnissen wurden die Waldflächen von Dedelow aus angefahren, wo sich die »Forschungsstation Landwirt- 


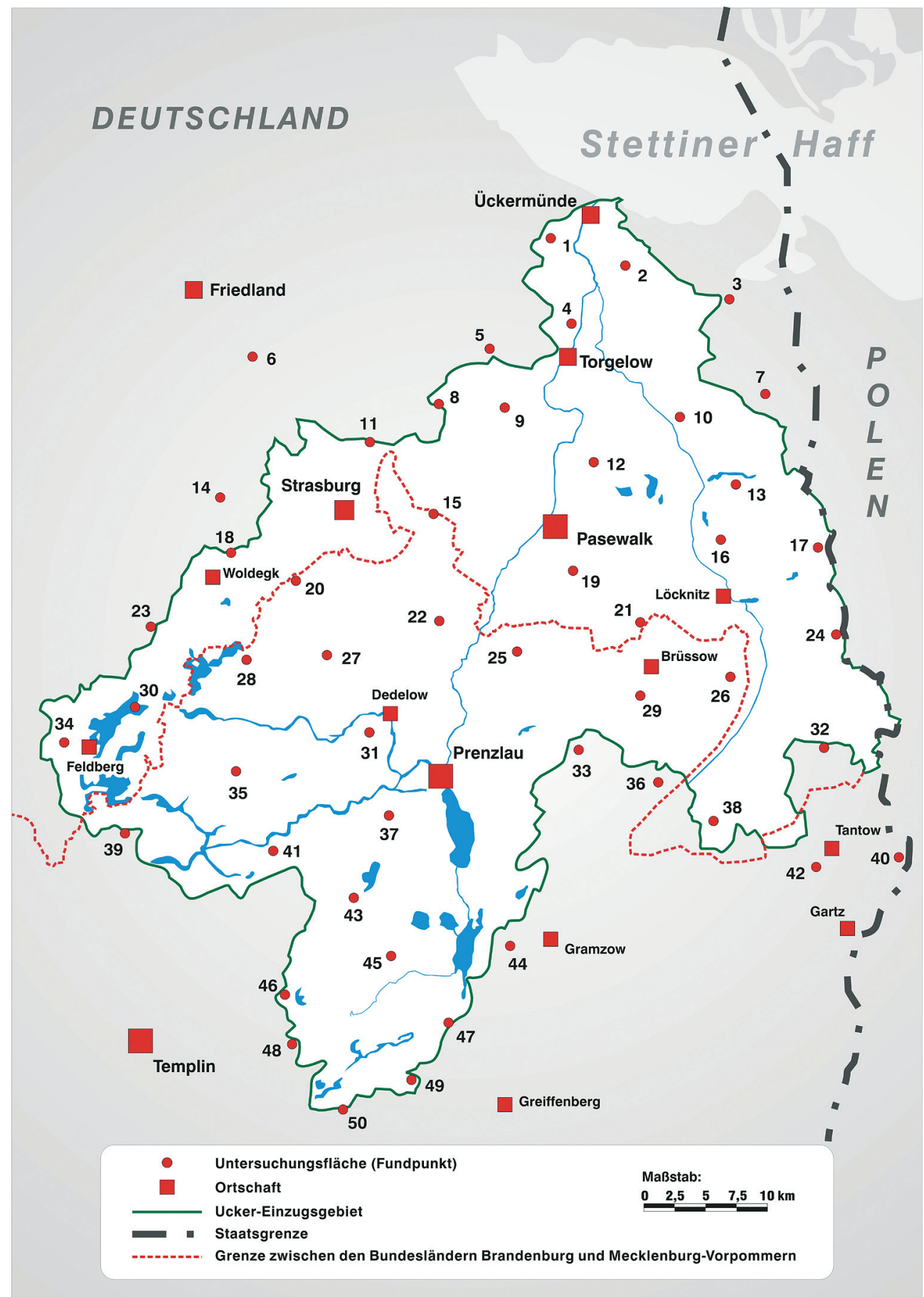

Fig. 1: Das Untersuchungsgebiet in der Uckermark mit den von 2001 bis 2003 besammelten Waldstandorten. Karte nach F. Menzel \& J. Kiesel. 
schaft" des Zentrums für Agrarlandschaftsforschung (ZALF) befindet. Die Aufsammlungen fanden im Rotationsprinzip drei Mal jährlich statt, so dass jede Fläche einmal zum Frühjahrs-, Sommer- und Herbstaspekt beprobt wurde. Für den gesamten Untersuchungszeitraum (20012003) ergab das einen Materialumfang von 150 Dipterenproben. Die Hippoboscidae-Imagines wurden in $70 \%$ igen Alkohol konserviert und in der Dipterensammlung des Senckenberg Deutschen Entomologischen Institutes (SDEI) in Müncheberg deponiert.

\section{Ergebnisse}

Insgesamt wurden an 20 verschiedenen Fundorten geflügelte Weibchen von Lausfliegen nachgewiesen (leg. F. Menzel; det. M. Jentzsch). Davon liegen neun im Bundesland Brandenburg und elf in Mecklenburg-Vorpommern. Nachstehend werden alle Hippoboscidenfunde aus dem Ucker-Einzugsgebiet bekanntgegeben.

\section{Hippobosca equina LinNAEUs, 1758}

BRANDENBURG: 1 ㅇ, Suckower Forst bei Friedrichsfelde W von Steinhöfel, 5305'24"N $13^{\circ} 50^{\prime} 15^{\prime \prime E}$ (Fundpunkt 49), $82 \mathrm{~m}$ ü. NN, Nadelwald (Kiefer) mit Birke und Holunder, 30.05.2003, leg. Menzel.

\section{Lipoptena cervi (LINNAEUS, 1758)}

(Fig. 2)

BRANDENBURG: 2 우, E von Göritz, 532 $24^{\prime} 45^{\prime \prime} \mathrm{N} 13^{\circ} 57^{\prime} 28^{\prime \prime E}$ (Fundpunkt 25), $45 \mathrm{~m}$ ü. NN, Laubwald (Hainbuche, Esche, Winterlinde, Ulme, Eberesche, Rotbuche, Weißdorn), 26.09.2002, leg. Menzel; 1 ㅇ, Hornshagen E von Woldegk, 5328'02"N 1341'39"E (Fundpunkt 20), 103 m ü. NN, Laubwald (Rotbuche, Stieleiche), 24.09.2003, leg. Menzel; 4 우 웅 Neuhof NW von Wilsickow, 5330'49"N 1350'44"E (Fundpunkt 15), $48 \mathrm{~m}$ ü. NN, Mischwald (Stieleiche, Traubeneiche, Lärche) mit Traubenkirsche, Eberesche und Hainbuche, 25.09.2001, leg. Menzel; 3 우 옹 Prenzlauer Stadtforst bei der Rummelpforter Mühle SE von Boitzenburg, $53^{\circ} 15^{\prime} 21^{\prime \prime N} 13^{\circ} 38^{\prime} 55^{\prime \prime E}$ (Fundpunkt 41), 83 m ü. NN, Nadelwald (Kiefer) mit Stieleiche, Birke, Rotbuche, Holunder und Eberesche, 25.09.2003, leg. Menzel; 1 ㅇ , Prenzlauer Stadtforst

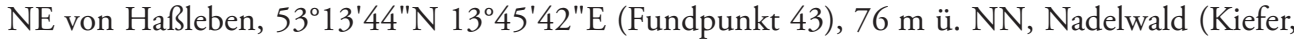
Fichte) mit Rotbuche, Bergahorn, Stieleiche, Haselnuß und Holunder, 26.07.2002, leg. Menzel; 1 우, Siedlung Moor S von Brüssow, 5322'54"N 1406'33"E (Fundpunkt 29), $62 \mathrm{~m}$ ü. NN, Nadelwald (Kiefer) mit Birke, Eberesche, Stieleiche und Traubenkirsche, 25.09.2002, leg. Menzel; 2 우 옹 Suckower Forst bei Friedrichsfelde W von Steinhöfel, 5305'24"N 1350'15"E (Fundpunkt 49), $82 \mathrm{~m}$ ü. NN, Nadelwald (Kiefer) mit Birke und Holunder, 29.09.2002, leg. Menzel; 2 우, Thomsdorf W von Boitzenburg, 5316'21"N 1328'47"E (Fundpunkt 39), 117 m ü. NN, Laubwald (Rotbuche), 24.09.2003, leg. Menzel; 1 ㅇ Randowbruch bei Wollschow

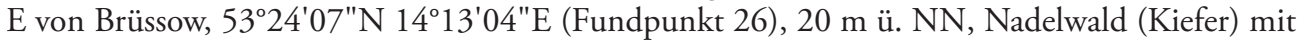
Stieleiche und Eberesche, 25.09.2002, leg. Menzel.

MECKLENBURG-VORPOMMERN: 1 ㅇ, Ahlbeck E von Eggesin, 5340'38"N 14²12'41"E (Fundpunkt 3), $3 \mathrm{~m}$ ü. NN, Nadelwald (Kiefer) mit Birke, 27.09.2001, leg. Menzel; 1 q , Borken

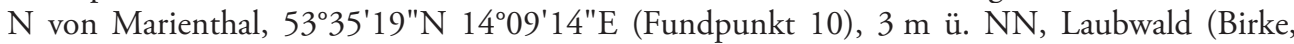
Echter Kreuzdorn, Esche), 28.09.2001, leg. Menzel; 1 웅 Caselow S von Rossow, 53²6'17"N 


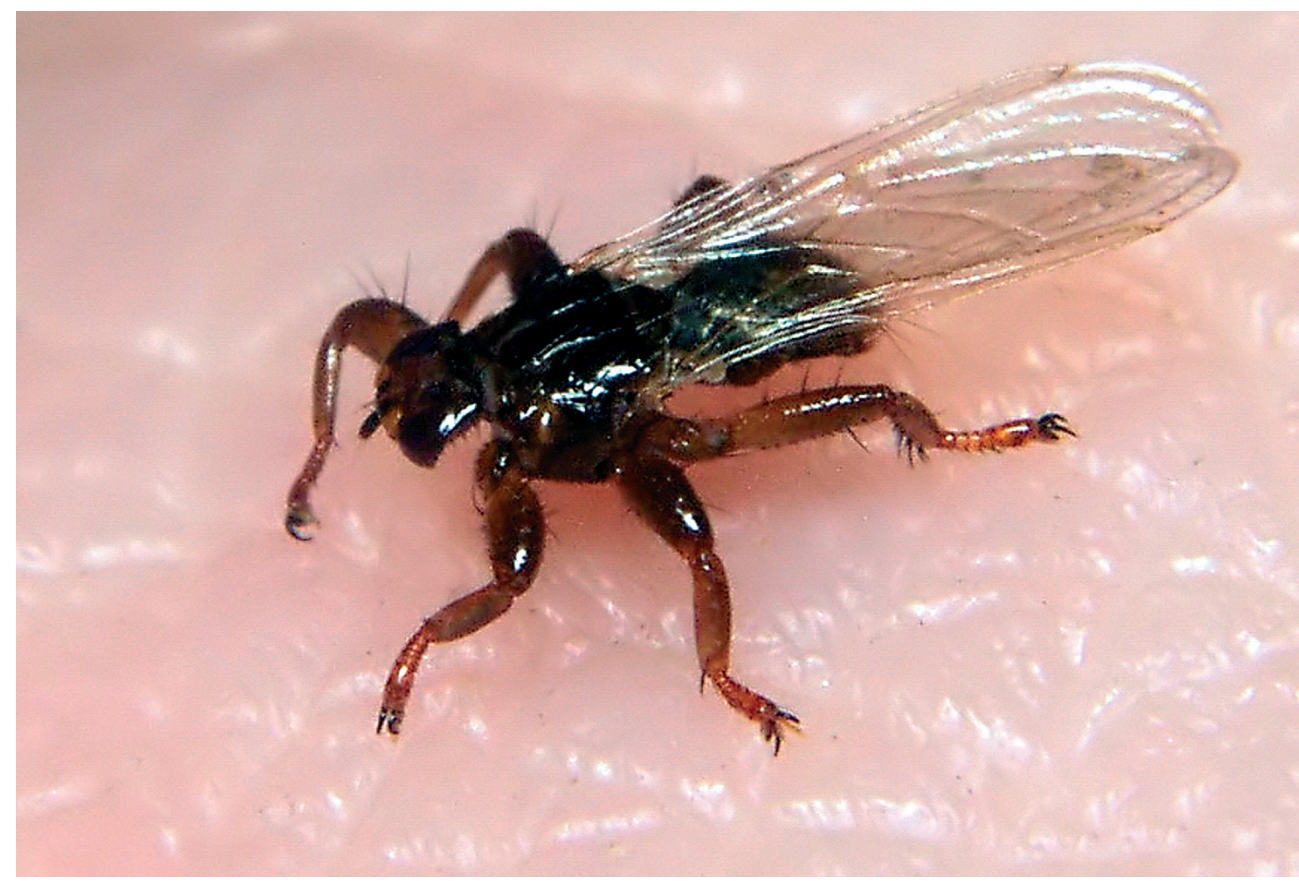

Fig. 2: Lipoptena cervi (Linnaeus) - Funddaten: Mecklenburg-Vorpommern, Dobbin SW von Dobbertin, Forst Barkhorst, Juni 2004). Foto: F. Koehler.

$14^{\circ} 06^{\prime} 25^{\prime} \mathrm{E}$ (Fundpunkt 21), $53 \mathrm{~m}$ ü. NN, Laubwald (Rotbuche, Hainbuche, Stieleiche),

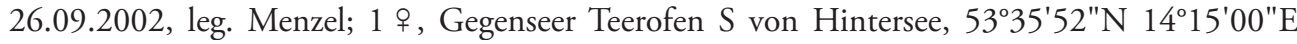
(Fundpunkt 7), $10 \mathrm{~m}$ ü. NN, Mischwald (Kiefer, Fichte, Rotbuche, Traubeneiche) mit ErlenBirken-Bruch, 27.09.2001, leg. Menzel; 9 ㅇ ㅇ , Gorkow N von Löcknitz, 53²9'14"N 14²12'21"E (Fundpunkt 16), $11 \mathrm{~m}$ ü. NN, Mischwald (Kiefer, Stieleiche, Birke), 26.09.2002, leg. Menzel; 2 우 우, Groß Miltzow E von Strasburg, 5331'22"N 13³5'43"E (Fundpunkt 14), 87 m ü. NN, Laubwald (Stieleiche, Bergahorn, Esche, Zwischenlinde, Birke, Holunder), 24.09.2001, leg.

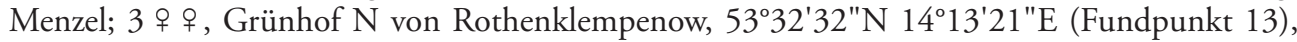
$5 \mathrm{~m}$ ü. NN, Nadelwald (Kiefer, Fichte), 27.09.2001, leg. Menzel; 9 우 Blankensee, 5329'14"N 14ำ'19"E (Fundpunkt 17), 31 m ü. NN, Mischwald (Kiefer, Fichte, Stieleiche, Birke, Eberesche), 28.09.2002, leg. Menzel; 1 ㅇ, N von Jatznick, 5335'29"N $13^{\circ} 56^{\prime} 04 " \mathrm{E}$ (Fundpunkt 9), $25 \mathrm{~m}$ ü. NN, Laubwald (Rotbuche, Traubeneiche) mit ErlenBirken-Bruch, 26.09.2001, leg. Menzel; 1 \% , Nettelgrund SE von Rothemühl, 533'ㄱ'38"N 1350'53"E (Fundpunkt 8), $23 \mathrm{~m}$ ü. NN, Mischwald (Rotbuche, Kiefer); 25.09.2001, leg.

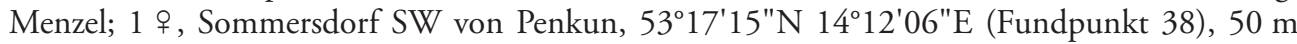
ü. NN, Nadelwald (Kiefer, Fichte, Lärche) mit Rotbuche, Bergahorn, Spitzahorn, Hainbuche und Winterlinde, 27.09.2002, leg. Menzel.

\section{Diskussion}

Mit den vorstehenden Untersuchungsergebnissen wird ein Beitrag zur Lausfliegen-Fauna Brandenburgs und Mecklenburg-Vorpommerns geleistet. Wenngleich zu vermuten ist, dass man noch mehr Präparate in musealen Einrichtungen oder Privatsammlungen auffinden kann, 
so existiert für beide Bundesländer noch keine flächendeckende Erfassung des Arteninventars. In der Literatur findet man für die Gebiete nur vereinzelte faunistische Angaben. So hat z. B. MüLler (2000a) die von Carl Friedrich Ketel in Mecklenburg-Vorpommern zusammengetragenen Hippobosciden ausgewertet. Neben Lepoptena cervi (Linnaeus, 1758) und Melophagus ovinus (Linnaeus, 1758) erwähnt er auch einen Fund von Ornithomya avicularia (Linnaeus, 1758) aus Woldegk (Funddatum: Juli 1898). Die Kleinstadt Woldegk (533' $\left.30^{\prime \prime N} 13^{\circ} 35^{\prime} 00^{\prime \prime E}\right)$ befindet sich am Rand des Ucker-Einzugsgebietes und liegt nur $3 \mathrm{~km}$ südwestlich von Fundpunkt 18 (Helpter Berge). In dem von uns ausgewerteten Material kam Ornithomya avicularia nicht vor. Als stationärer Ektoparasit auf Vögeln (WALTER 1990) und wegen der angewandten Sammeltechnik (bodennaher Kescherfang) war diese Art in den Proben auch kaum zu erwarten. Raddatz (1873) führt für Mecklenburg-Vorpommern ebenfalls die drei bei MüLLER (2000a) gemeldeten Spezies an und listet zusätzlich noch die beiden Arten Stenepteryx hirundinis (Linnaeus, 1758) sowie Hippobosca equina Linnaeus, 1758 auf. An anderer Stelle dokumentierte Frank Koehler (Bornheim) einen bisher unpublizierten Fund von Lipoptena cervi in Mecklenburg-Vorpommern (Fig. 2). Er fotografierte im Juni 2004 ein Weibchen im Forst Barkhorst bei Dobbin [nahe Dobbertin, ca. 22 km SW von Güstrow], das von Joachim Müller (Magdeburg) determiniert wurde. Weitere Nachweise hat Lange (2008) veröffentlicht. Im Landkreis Parchim fand er Hippobosca equina am Rande des NSG "Marienfließ "südlich von Retzow und im NSG "Quasliner Moor" bei Wahlsdorf (beide Funde am 06.07.2008). Die Art wurde auch am 31.07.2008 bei Buschhof (Landkreis Müritz) registriert. In der gleichen Publikation weist der Autor noch auf eine ältere Beobachtung vom 19.06.2007 im Heidegebiet zwischen Retzower Wald und NSG »Marienfließ « hin (LANGE \& LüPKE 2007).

Für die Mark Brandenburg - die im 19. Jahrhundert über das heutige Staatsgebiet hinaus weit nach Polen hineinreichte - hat NeuHaus (1886) die Hippobosciden-Funde zusammengestellt. Sein Hauptsammelgebiet schloß die Städte Freienwalde [= Bad Freienwalde], Liebenwalde und Oranienburg ein. NeuHaus bestimmte aber auch Lausfliegen von anderen Entomologen und berücksichtigte in der "Diptera Marchica" Zweiflügler aus der Mark, deren genaue Herkunft nicht überliefert ist. Den einzigen konkreten Bezug zum heutigen Bundesland Brandenburg weist ein Fund von Lipoptena cervi auf ( $\sigma^{\star}$ und 9 im Schloßgarten von Oranienburg). Ob die anderen Bestände in der Sammlung NeuHaus von Hippobosca equina (4 Exemplare), Ornithomya avicularia (2 Exemplare), Stenepteryx hirundinis (4 Exemplare) und Melophagus ovinus (2 Exemplare) das heutige Bundesland Brandenburg betreffen, bleibt unklar. Als sicher gilt jedoch, dass die dort niedergeschriebenen Nachweise mit der Bezeichnung „Berlin (Ruthe)“ [= Berlin, leg./coll. Ruthe] entweder dem Bundesland Berlin oder den früheren preußischen Provinzen Posen und Schlesien in Polen zuzurechnen sind [siehe Vorwort in Neunaus (1886)]. Somit scheidet ein bei Neunaus (1886: 346) aufgelisteter Fund der Mauerseglerlausfliege Crataerina pallida (LATreILle, 1811) für das von uns betrachtete Faunengebiet aus. Ebenfalls für die Mark Brandenburg werden von MAA (1969: 4) - auf den sich auch Müller (1997: 117) stützt - 2 o $^{\top}$ o $^{\star}$ und 3 ㅇ ㅇ von Ornithophila metallica (Schiner, 1864) erwähnt. Diese Fundmeldung bezieht sich jedoch auf die Lokalität "Charlottenburg" - einem Stadtteil von Berlin, der nicht dem Bundesland Brandenburg zuzurechnen ist. Darüber hinaus liegen aus dem Bundesland Brandenburg noch zwei unpublizierte Hippobosciden-Funde vor, die wir an dieser Stelle bekanntgeben möchten. So wurden am 06.06.2009 auf einer Exkursion im Roten Luch südwestlich von Waldsieversdorf [Naturpark

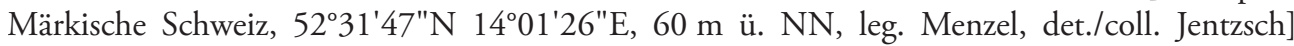
zwei 우 9 von Lipoptena cervi in einem Stieleichen-Hochwald gefangen. Außerdem fand W. Bäse am 31.07.1989 ein $0^{*}$ von Ornithomya avicularia in Belzig [52 08'30"N 12³5'39"E, det./coll. Jentzsch]. Folglich setzt sich die Hippobosciden-Fauna in Brandenburg zur Zeit aus 3 und in Mecklenburg-Vorpommern aus 5 Lausfliegenarten zusammen (Tabelle 1). 
Mit sehr wenigen Ausnahmen gelangen die hier vorgestellten Nachweise der auf Säugetieren parasitierenden Hirschlausfliegen Ende September, also einer Zeit, in der die Tiere als flugfähige Imagines auf der Suche nach ihren Hauptwirten - den Rehen - häufig anzutreffen sind. Die Tiere werfen erst nach Erreichen ihres Wirtes die Flügel ab, um dann die kurz vor der Verpuppung stehenden Larven (Pupiparie) im Fell der Wirte abzusetzen. Dies könnte ein Grund dafür sein, dass ausnahmslos Weibchen gefangen wurden, weil diese möglichst rasch einen Wirt finden und entsprechende Aktivitätsradien entfalten müssen. Hinzu kommt, dass die männlichen Lausfliegen in der Regel kleiner und schwieriger zu fangen sind (BütTIKeR 1994). Bei den Untersuchungsflächen, auf denen die Hippobosciden gefunden wurden, handelt es sich ausnahmslos um Laub- oder Mischwälder. Diese Lebensräume bieten den Rehen auch tagsüber genügend Deckung. Bei dem Einzelnachweis von Hippobosca equina wird vermutet, dass das Tier von einer Pferdekoppel in der Ortslage Friedrichsfelde stammt, die sich in südöstlicher Richtung vom beprobten Kiefernforst (Fundpunkt 49) anschließt.

Tab. 1: Lausfliegen-Nachweise aus den Bundesländern Mecklenburg-Vorpommern und Brandenburg. Legende: $\mathrm{BB}=$ Brandenburg; $\mathrm{MV}=$ Mecklenburg-Vorpommern .

\begin{tabular}{|c|c|c|c|}
\hline Art & Literaturquelle & $\mathrm{BB}$ & MV \\
\hline $\begin{array}{l}\text { Hippobosca equina } \\
\text { LinNaEus, } 1758\end{array}$ & $\begin{array}{l}\text { Jentzsch \& MenZel (vorliegende Daten) } \\
\text { RAdDATZ (1873); LANGE \& LÜPKe (2007); LANGE (2008) }\end{array}$ & $\sqrt{ }$ & $\sqrt{ }$ \\
\hline $\begin{array}{l}\text { Lipoptena cervi } \\
\text { (LinnaEus, 1758) }\end{array}$ & $\begin{array}{l}\text { Neuhaus (1886); Jentzsch \& Menzel (vorliegende Daten) } \\
\text { RadDatz (1873); MÜller (2000a); Jentzsch \& Menzel } \\
\text { (vorliegende Daten) }\end{array}$ & $\sqrt{ }$ & $\sqrt{ }$ \\
\hline $\begin{array}{l}\text { Melophagus ovinus } \\
\text { (LinnaEus, 1758) }\end{array}$ & RAdDATZ (1873); MÜLLER (2000a) & & $\sqrt{ }$ \\
\hline $\begin{array}{l}\text { Ornithomya avicularia } \\
\text { (LinNAEUs, 1758) }\end{array}$ & $\begin{array}{l}\text { Jentzsch \& Menzel (vorliegende Daten) } \\
\text { RadDAtz (1873); Müller (2000a) }\end{array}$ & $\sqrt{ }$ & $\sqrt{ }$ \\
\hline $\begin{array}{l}\text { Stenepteryx hirundinis } \\
\text { (LinNAEUs, 1758) }\end{array}$ & RADDATZ (1873) & & $\sqrt{ }$ \\
\hline
\end{tabular}

\section{Danksagung}

Wir bedanken uns recht herzlich bei Herrn Frank Köhler (Bornheim), der uns das Foto von Lipoptena cervi (Linnaeus) freundlicherweise zur Verfügung gestellt hat. Bei der Erarbeitung und Umsetzung der Karte haben uns Joachim Kiesel (Institut für Landschaftssystemanalyse, ZALF e. V. Müncheberg) und Bianka Katnig (Eberswalde) unterstützt. Außerdem bedankt sich der Zweitautor beim Zentrum für Agrarlandschaftsforschung (ZALF) e.V. für die finanzielle Unterstützung bei der Durchführung der Freilandarbeiten sowie für die Möglichkeit der kostenlosen Benutzung von Räumlichkeiten in der "Forschungsstation Landwirtschaft» in Dedelow (Leiter: Dr. Gernot Verch).

\section{Literatur}

Büttiker, W. 1994: Die Lausfliegen der Schweiz (Diptera, Hippoboscidae). - Documenta Faunistica Helvetiae 15: 1-117.

Kock, D. 2000: Ornithoica turdi (Latreille, 1812) neu in der Fauna Deutschlands und ihre Phänologie in der westlichen Paläarktis (Insecta: Diptera: Hippoboscidae). - Senckenbergiana biologica 80 (1-2): 155-158.

Lange, L. 2008: Funde von Pferdelausfliegen Hippobosca equina Linnaeus, 1758 in den mecklenburgischen Kreisen Parchim und Müritz (Diptera, Hippoboscidae). - Entomologische Nachrichten und Berichte 52 (3-4): 214. 
Lange, L. \& Lüpke, M. 2007: Kontrolle künstlicher Nisthilfen für den Rauhfußkauz Aegolius funereus im Retzower Wald (Kreis Parchim, Mecklenburg-Vorpommern). - Vogelkundliche Berichte zwischen Küste und Binnenland 6: 97-98.

MAA, T. C. 1969: Synopsis of the genera Ornithophila and Ornithoctona with remarks on their habitat diversification (Diptera: Hippoboscidae). [1-23] - In MAA, T. C.: Studies in Hippoboscidae (Diptera). Part 2. - Pacific Insects Monograph 20: 1-312.

MülleR, J. 1985: Erstnachweis der Hirschlausfliege Lipoptena cervi L., 1758 auf Damwild Dama dama (L., 1758) in der Altmark, DDR-Bezirk Magdeburg. - Abhandlungen und Berichte für Naturkunde und Vorgeschichte 12 (6): 55-58.

MüLleR, J. 1989: Neuere Ektoparasitenfunde (Diptera: Hippoboscidae, Nycteribiidae; Siphonaptera) von Wildvögeln und Wildsäugern und deren hygienische Bedeutung. [366-370] - In: KLAUsNitzer, B. \& Dunger, W. (Hrsg.): Verhandlungen des elften internationalen Symposiums für die Entomofaunistik Mitteleuropas (SIEEC), 19.-23. Mai 1986, Gotha: 1-398.

MüLLER, J. 1997: Lausfliegen-Funde von heimischen Vögeln, nebst Bemerkungen zur deutschen Checkliste Diptera: Hippoboscidae. - Ornithologische Jahresberichte des Museum Heineanum 15: 115-132.

Müller, J. 1999: Hippoboscidae. [155-156] - In: Schumann, H.; Bährmann, R. \& Stark, A. (Hrsg.): Entomofauna Germanica 2. Checkliste der Dipteren Deutschlands. - Studia dipterologica Supplement 2: $1-354$.

Müller, J. 2000a: Hippoboscidae. [193-194] - In: Ziegler, J. \& Menzel, F. (Hrsg.): Die historische Dipteren-Sammlung Carl Friedrich KeTEL. Revision einer zwischen 1884 und 1903 angelegten Sammlung von Zweiflüglern (Diptera) aus Mecklenburg-Vorpommern. - Nova Supplementa Entomologica 14: $1-266$.

MüLleR, J. 2000b: Crataerina melbae auf Alpenseglern Tachymarpis melba in Freiburg im Breisgau als Ergänzung zur deutschen Checkliste der Hippoboscidae (Diptera). - Studia dipterologica 7 (2): 501-505.

MÜLlER, J. 2009: Lausfliegen - Hippoboscidae. - Website http://www.faunoekjmueller-magdeburg.de/ Lausfliegen/body_lausfliegen.html [Download 7.7.2009].

Müller, J. \& Schmidt, M. 2000: Nachweis von Crataerina melbae (Diptera: Hippoboscidae) auf Alpenseglern Tachymarptis melba in Freiburg im Breisgau. - Ornithologische Jahresberichte des Museum Heineanum 18: 129-138.

Neuhaus, G. H. 1886: Diptera Marchica. Systematisches Verzeichniss der Zweiflügler (Mücken \& Fliegen) der Mark Brandenburg. Mit kurzer Beschreibung und analytischen Bestimmungs-Tabellen. - Berlin: Nicolaische Verlags-Buchhandlung R. Stricker: i-xvi + 1-371 + Tafel I-VI.

Raddatz, A. 1873: Uebersicht der in Mecklenburg bis jetzt beobachteten Fliegen (Diptera). - Archiv des Vereins der Freunde der Naturgeschichte in Mecklenburg 27: 22-131.

Schmidt, E. 2001: Nachweis der Reiherlausfliege Icosta ardeae (MACQUART, 1835) (Diptera, Hippoboscidae) in Thüringen. - Anzeiger des Vereins Thüringer Ornithologen 4: 233-234.

WALter, G. 1990: Dipteren (Diptera: Cyclorrapha) als Ektoparasiten von Vögeln in der Bundesrepublik Deutschland. - Die Vogelwarte 35: 231-242.

Anschrift der Verfasser:

Dr. Matthias Jentzsch

Schillerstraße 35

06114 Halle (Saale)

Germany

E-mail: m_jentzsch@yahoo.de
Dr. Frank Menzel

Senckenberg Deutsches Entomologisches Institut

Eberswalder Straße 90

15374 Müncheberg

Germany

E-mail: frank.menzel@senckenberg.de
Subject editor:

Dr. D. Werner 